\title{
CPAP and measures of cardiovascular risks in patients with OSAS
}

\section{To the Editors:}

We read with great interest the article by KOHLER et al. [1], which was published in a previous issue of the European Respiratory Journal. In their study, the authors emphasized that continuous positive airway pressure (CPAP) treatment can reduce the cardiovascular risks by reducing sympathetic nerve activity, ambulatory blood pressure and arterial stiffness, and increasing sensitivity of the arterial baroreflex in sleep apnoea patients.

The pathogenesis of cardiovascular disease in obstructive sleep apnoea syndrome (OSAS) is not completely understood but is likely to be multifactorial, involving a diverse range of mechanisms, including sympathetic nervous system overactivity, selective activation of inflammatory molecular pathways, endothelial dysfunction, abnormal coagulation and metabolic dysregulation, with the latter particularly involving insulin resistance and disordered lipid metabolism [2].

CPAP not only directly reduces the cardiovascular risks in sleep apnoea patients but can also indirectly facilitate weight loss with different metabolic events, such as reducing insulin resistance [3]. However, after CPAP use in OSAS patients, CoUGHLIN et al. [4] reported no change in glucose, lipids, insulin resistance or the proportion of patients with metabolic syndrome. In CPAPcompliant patients, the fall in blood pressure was greater and the baroreceptor sensitivity improved significantly but no metabolic variable changed. In obese Caucasians with untreated obstructive sleep apnoea, CPAP can improve baroreceptor responsiveness and reduce waking blood pressure within 6 weeks, but this treatment period was insufficient to modify insulin resistance or change the metabolic profile [4].

We would like to emphasise that the factors that might account for the reduction of cardiovascular risk in OSAS patients with the use of CPAP as reported by KOHLER et al. [1] are complex and need to be further studied to better understand the underlying mechanisms of this disease.

\section{Enoz* and H.M. Inancli ${ }^{*}$}

*Dept of ORL \& Head and Neck Surgery, Special Yenibosna Safa Hospital, Istanbul, and "Dept of ORL \& Head and Neck Surgery, Ankara Oncology Training and Research Hospital, Ankara, Turkey.

\section{STATEMENT OF INTEREST}

None declared.

\section{REFERENCES}

1 Kohler M, Pepperell JC, Casadei B, et al. CPAP and measures of cardiovascular risk in males with OSAS. Eur Respir J 2008; 32: 1488-1496.

2 McNicholas WT, Bonsigore MR. Management Committee of EU COST ACTION B26. Sleep apnoea as an independent risk factor for cardiovascular disease: current evidence, basic mechanisms and research priorities. Eur Respir J 2007; 29: 156-178.

3 Schahin SP, Nechanitzky T, Dittel C, et al. Long-term improvement of insulin sensitivity during CPAP therapy in the obstructive sleep apnoea syndrome. Med Sci Monit 2008; 14: CR117-CR21.

4 Coughlin SR, Mawdsley L, Mugarza JA, et al. Cardiovascular and metabolic effects of CPAP in obese males with OSA. Eur Respir J 2007; 29: 720-727.

DOI: $10.1183 / 09031936.00001809$ 